\title{
What are considerations for neonates at risk for COVID-19?
}

\author{
Soo-Han Choi, MD, PhD
}

Department of Pediatrics, Hallym University Dongtan Sacred Heart Hospital, Hwaseong, Korea

The World Health Organization (WHO) declared the coronavirus disease 2019 (COVID-19) outbreak caused by severe acute respiratory syndrome coronavirus 2 (SARS-CoV-2) a pandemic on March 11, 2020. As of mid-June 2020, over 8 million cases have been confirmed globally. ${ }^{1}$ However, data are limited on the transmission, incidence, and effect of SARS$\mathrm{CoV}-2$ infection in mothers and newborns.

A recent WHO report noted that, of 115 mother-infant pairs from 17 studies in which the mother had confirmed COVID-19, 13 infants had COVID-19.2) Of the 20 whose breastmilk was tested for SARS-CoV-2 RNA particles by reverse transcriptionpolymerase chain reaction (RT-PCR), 18 were negative and 2 were positive; one mother's baby was not infected with COVID-19 (mix-fed), while the other was infected (feeding modality not reported). Knight et al. ${ }^{3)}$ reported the United Kingdom national cohort study of 427 pregnant women admitted to the hospital with confirmed SARS-CoV-2 infection. The estimated incidence of the hospital admission of pregnant women with confirmed SARS-CoV-2 infection was 4.9 (95\% confidence interval, 4.5-5.0). ${ }^{4)}$ per 1,000 pregnant women. Four women had a miscarriage at 10-19 weeks' gestation. Of the 262 women who gave birth, 66 (25\%) did so preterm: $32(48 \%)$ due to maternal COVID-19, 9 (14\%) due to fetal compromise, and $12(18 \%)$ due to other obstetric conditions. Three neonates were stillborn and 2 died in the neonatal period; neither of the neonatal deaths was attributed to SARS-CoV-2. Twelve of the newborns (5\%) tested positive for SARS-CoV-2 RNA: 6 within the first 12 hours of life. Two of 6 infants with early-onset infection (positive test at $<12$ hours of age) were born vaginally, while the other 4 were born by caesarean ( 3 prelabor). In cases of late-onset infection (positive test at $\geq 12$ hours of age), 4 were prelabor caesarean and 2 were vaginal births. Viral analyses were not performed of the umbilical cord blood, placenta, or vaginal secretions.

Current evidence is inconclusive about the transplacental viral transmission from mother to newborn. ${ }^{2-5)}$ SARS-CoV-2 transmission to neonates is thought to occur primarily through respiratory droplets during the postnatal period when neonates are exposed to mothers, other caregivers, visitors, or healthcare personnel with COVID-19.4) Since maternal and newborn care units vary in physical configuration, each facility should consider the appropriate space and staffing needs to prevent SARS-CoV-2 transmission. These considerations include the appropriate isolation of pregnant patients with suspected or confirmed COVID-19 and their babies, training for all healthcare personnel on those units to include appropriate infection control practices and personal protective equipment use and handling, and appropriate SARS-CoV-2 testing of newborn infants. ${ }^{5)}$

RT-PCR testing of upper respiratory tract specimens is generally recommended for all neonates born to women with confirmed or suspected COVID-19 regardless of whether the neonate has signs of infection. However, the optimal timing for testing after birth is unknown. Early testing may lead to false positives (due to contamination by SARS-CoV-2 RNA from maternal fluids) or false negatives (virus not detectable immediately after exposure following delivery). Newborns should be bathed after birth to remove any virus potentially present on the skin surface. However, SARS-CoV-2 antibody tests are not recommended for detecting current infection in neonates. ${ }^{2,4,6}$

Guidance on perinatal care of newborns whose mothers have suspected or confirmed COVID-19 were provided by the WHO, the US Centers for Disease Control and Prevention, and the American Academy of Pediatrics (AAP).,2,4) The 3 organizations suggest that the benefits of breastfeeding in the setting of COVID-19 appear to outweigh the potential risks of viral transmission from mother to infant. However, there are some differences among their guidelines in breastfeeding methods as well as maternal and newborn separation practices (Table 1). ${ }^{2,6,7)}$ The WHO recommends that an infected mother is in close contact with her baby; in infants, the risk of SARS-CoV-2 infection is low, the infection is typically mild or asymptomatic, and the consequences of not breastfeeding or of separating mother and child can be significant. ${ }^{2}$ The AAP focuses on minimizing neonatal infection by suggesting that the risks and benefits of temporary maternal-newborn separation be discussed with the mother by the healthcare team and decisions be made in accordance with the mother's wishes. ${ }^{6}$ These guidelines are being updated as more clinical information and scientific evidence become available.

The city of Daegu had the first large outbreak of COVID-19

Corresponding author: Soo-Han Choi, MD, PhD. Division of Pediatric Infectious Diseases, Department of Pediatrics, Hallym University Dongtan Sacred Heart Hospital, 7 , Keunjaebong-gil, Hwaseong 18450, Korea 
Table 1. Comparison of recent recommendations for newborns whose mothers have suspected or confirmed COVID-19

\begin{tabular}{|c|c|c|c|}
\hline & $\mathrm{WHO}^{2)}$ & $A A P^{6)}$ & $U^{7)}$ \\
\hline Use of delayed-cord clamping & Recommended & Should continue per usual center practice & Not mentioned \\
\hline Mother-neonatal contact & Strongly recommended & Recommended temporary separation ${ }^{a)}$ & $\begin{array}{l}7 \text { Days after the onset of mother's } \\
\text { symptoms if she is well }\end{array}$ \\
\hline Breastfeeding & Recommended & Recommended ${ }^{\text {b) }}$ & Recommended \\
\hline Timing of testing for SARS-CoV-2 & Not mentioned & $\begin{array}{l}\text { First at approximately } 24 \text { hours of age and } \\
\text { again at approximately } 48 \text { hours of age } \\
\text { A single test at } 24-48 \text { hours of age (discharged } \\
\text { prior to } 48 \text { hours of age) }\end{array}$ & At 72 hours and again on day $5^{\text {c) }}$ \\
\hline
\end{tabular}

COVID-19, coronavirus disease 2019; WHO, World Health Organization; AAP, American Academy of Pediatrics; UK, United Kingdom; SARS-CoV-2, severe acute respiratory syndrome coronavirus 2 .

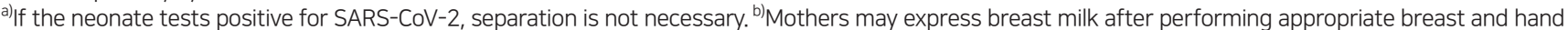
hygiene and the infant may be fed by other uninfected caregivers. ${ }^{c}$ There is no indication for routine testing of asymptomatic babies, but it can be performed if resources permit.

outside of China in late February to March 2020. Predominance by the 20 s age group and female sex may have caused by the outbreak related to a religious group in Daegu. ${ }^{8)}$ Lee et al. ${ }^{9}$ reported the case of the first newborn baby born to a mother with confirmed COVID-19 in Daegu, South Korea. At that time, the guidance on perinatal care started with extremely little evidence. ${ }^{10)}$ The situation of COVID-19 in Korea is still evolving; therefore, it is imperative to properly update the guidelines for COVID-19 in a timely manner.

\section{Conflicts of interest}

No potential conflicts of interest relevant to this article are reported.

See the article "Management of the first newborn delivered by a mother with COVID-19 in South Korea" via https://doi. org/10.3345/cep.2020.00850.

\section{References}

1. World Health Organization. Coronavirus disease (COVID-19) pandemic [Internet]. Geneva (Switzerland): World Health Organization; [cited 2020 Jun 20]. Available from: https://www.who.int/emergencies/diseases/ novel-coronavirus-2019.

2. World Health Organization. Clinical management of COVID-19: Interim guidance 27 May 2020 [Internet]. Geneva (Switzerland): World Health Organization; [cited 2020 Jun 20]. Available from: https://www.who.int/ publications/i/item/clinical-management-of-covid-19.

3. Knight M, Bunch K, Vousden N, Morris E, Simpson N, Gale C, et al. Characteristics and outcomes of pregnant women admitted to hospital with confirmed SARS-CoV-2 infection in UK: national population based cohort study. BMJ 2020;369:m2107. https://doi.org/10.1136/bmj.m2107.

4. Centers for Disease Control and Prevention. Coronavirus Disease 2019 (COVID-19). Evaluation and management considerations for neonates at risk for COVID-19 [Internet]. Atlanta (GA): Centers for Disease Control and Prevention; [cited 2020 Jun 20]. Available from: https://www.cdc. gov/coronavirus/2019-ncov/hcp/caring-for-newborns.html.

5. Centers for Disease Control and Prevention. Coronavirus Disease 2019 (COVID-19). Considerations for inpatient obstetric healthcare settings [Internet]. Atlanta (GA): Centers for Disease Control and Prevention; [cited 2020 Jun 20]. Available from: https://www.cdc.gov/coronavirus/ 2019-ncov/hcp/inpatient-obstetric-healthcare-guidance.html.

6. American Academy of Pediatrics. FAQs: management of infants born to mothers with suspected or confirmed COVID-19 [Internet]. Itasca (IL): American Academy of Pediatrics; c2020 [updated 2020 Jul 22; cited 2020 Jun 20]. Available from: https://services.aap.org/en/pages/2019-novelcoronavirus-covid-19-infections/clinical-guidance/faqs-management-ofinfants-born-to-covid-19-mothers/.

7. British Association of Perinatal Medicine. COVID-19 pandemic. Frequently asked questions within neonatal services. A BAPM supplement to RCPCH guidance [Internet]. London: British Association of Perinatal Medicine; [updated 2020 Jun 10; cited 2020 Jun 20]. Available from: https://hubble-live-assets.s3.amazonaws.com/bapm/redactor2_assets/ files/550/COVID-FAQs_11.6.20.docx.pdf.

8. Korean Society of Infectious Diseases, Korean Society of Pediatric Infectious Diseases, Korean Society of Epidemiology, Korean Society for Antimicrobial Therapy, Korean Society for Healthcare-associated Infection Control and Prevention, Korea Centers for Disease Control and Prevention. Report on the Epidemiological Features of Coronavirus Disease 2019 (COVID-19) Outbreak in the Republic of Korea From January 19 to March 2, 2020. J Korean Med Sci 2020;35:e112.

9. Lee EK, Kim WD, Lee DW, Lee SA. Management of the first newborn given birth by a mother with COVID-19 in South Korea. Clin Exp Pediatr 2020;63:373-5.

10. Kim KH, Cho EY, Kim DH, Kim HW, Park JY, Eun BW, et al. Guidelines for coronavirus disease 2019 response in children and adolescents. Pediatr Infect Vaccine 2020;27:24-34. 